\title{
A note on commutators in the group of infinite triangular matrices over a ring
}

\author{
A. Bier ${ }^{a * *}$ and Waldemar Hołubowski ${ }^{\mathrm{a}}$ \\ ${ }^{a}$ Institute of Mathematics, Silesian University of Technology, \\ ul. Kaszubska 23, 44 - 100 Gliwice, Poland
}

November 23, 2015

\begin{abstract}
We investigate the commutators of elements of the group $\mathrm{UT}(\infty, R)$ of infinite unitriangular matrices over an associative ring $R$ with 1 and a commutative group $R^{*}$ of invertible elements. We prove that every unitriangular matrix of a specified form is a commutator of two other unitriangular matrices. As a direct consequence we give a complete characterization of the lower central series of the group $\mathrm{UT}(\infty, R)$ including the width of its terms with respect to basic commutators and Engel words. With an additional restriction on the ring $\mathrm{R}$, we show that the derived subgroup of $\mathrm{T}(\infty, R)$ coincides with the group $\mathrm{UT}(\infty, R)$. The obtained results generalize the results obtained for triangular groups over a field.
\end{abstract}

\section{Introduction}

Let $R$ be an associative ring with 1 and $R^{*}$ be its group of invertible elements. By $\mathrm{T}(\infty, R)$ (and $\mathrm{T}(n, R)$ ) we denote the group of upper triangular matrices indexed by $\mathbb{N} \times \mathbb{N}$ (of size $n \times n$, respectively), whose inverses are also upper triangular. The requirement on the inverses is substantial here, as the inverse of an upper triangular matrix is not necessarily an upper triangular matrix. Illustrative examples of upper triangular matrices over a noncommutative ring, whose inverses are lower triangular can be found in [1,2]. A triangular matrix $A$ (finite or infinite dimensional) is called unitriangular, if all its diagonal entries are equal to 1 . $\operatorname{By~} \mathrm{UT}(\infty, R)$ and $\mathrm{UT}(n, R)$ we denote the groups of respectively infinite $\mathbb{N} \times \mathbb{N}$ and finite $n \times n$ unitriangular matrices and by $\mathrm{UT}(\infty, m, R)$ and $\mathrm{UT}(n, m, R)$ we denote their subgroups containing exactly all these matrices, which have zero entries on the first $m$ superdiagonals.

The properties of matrix groups $\mathrm{T}(\infty, R), \mathrm{UT}(\infty, R)$ and their finite dimensional analogues have been intensively studied recently. We refer to $[3,4,5,6$,

**Corresponding author. Email: lagnieszka.bier@polsl.pl 
$7,8,9]$ (for infinite matrices) and $[11,12,13,14,15,16]$ (for finite matrices) and references therein.

In the following $\mathbf{1}_{\infty}$ denotes the infinite identity matrix, i.e. the diagonal matrix having ring unity 1 on the main diagonal. Similarly, $\mathbf{1}_{i, j}$ denotes the matrix (finite or infinite, depending on the context) with a unique nonzero entry equal to 1 in the place $(i, j)$. We also write $\mathbf{1}_{n}$ for an $n$ by $n$ identity matrix. The commutator $[x, y]$ of elements $x$ and $y$ from a group $G$ is defined as a product $[x, y]=x^{-1} y^{-1} x y$.

The main result of our paper concerns commutators in $\mathrm{UT}(\infty, R)$.

Basic Theorem Let $R$ be an associative ring with 1 , such that $R^{*}$ is commutative. Then every matrix $C \in \mathrm{UT}(\infty, m, R), m=1,2, \ldots$, is a commutator $C=[A, B]$ of a matrix $A \in \mathrm{UT}(\infty, m-1, R)$ and the matrix $B$, such that $B^{-1}=\mathbf{1}_{\infty}+\sum_{n=1}^{\infty} \mathbf{1}_{i, i+1}$.

We note that $\operatorname{ring} R$ with commutative $R^{*}$ need not to be commutative itself. The standard example of a noncommutative ring $R$ with commutative $R^{*}$ is the free associative algebra $K\langle x, y\rangle$ in noncommuting variables $x, y$ over a field $K$. For other examples we refer to [17, 18, 19, 20, 21].

The above theorem has few important consequences, which we discuss in detail in the last part of our paper. In particular, it has direct implications on the structure of the lower central series of groups $\mathrm{T}(\infty, R)$ and $\mathrm{UT}(\infty, R)$ and on the respective width of their terms.

The lower central series of a group $G$ is the (possibly infinite) series of subgroups

$$
G=\gamma_{1}(G) \geq \gamma_{2}(G) \geq \ldots,
$$

defined recursively with $\gamma_{i+1}(G)=\left[\gamma_{i}(G), G\right]$, where for two subgroups $H_{1}$ and $H_{2}$ of $G$ by $\left[H_{1}, H_{2}\right]$ we denote the subgroup generated by all commutators $\left[h_{1}, h_{2}\right]$ such that $h_{1} \in H_{1}, h_{2} \in H_{2}$ (see [22]). We may also regard these terms as subgroups generated by all values of the so-called basic commutators $c_{i}\left(x_{1}, x_{2}, \ldots, x_{i}\right)$, where $c_{1}\left(x_{1}\right)=x_{1}$ and $c_{i+1}\left(x_{1}, \ldots, x_{i+1}\right)=\left[c_{i}\left(x_{1}, \ldots, x_{i}\right), x_{i+1}\right]$ (a value of a word is obtained by substituting all letters by elements of $G$ and calculating the resulting element of $G$ ). The subgroup $\gamma_{2}(G)=[G, G]$ is called the derived subgroup of $G$.

We mention here that the lower central series of the group $\mathrm{UT}(\infty, R)$ in the case $R$ is a field $(|R|>2)$ is determined in [5]. Our Basic Theorem allows for generalization of this result to the group of infinite matrices over certain rings.

Corollary 1.1. If $R$ is an associative ring with 1 , such that $R^{*}$ is commutative, then the lower central series of the group $\mathrm{UT}(\infty, R)$ is the sequence of subgroups:

$$
\mathrm{UT}(\infty, R) \geq \mathrm{UT}(\infty, 1, R) \geq \mathrm{UT}(\infty, 2, R) \geq \ldots,
$$

where $\gamma_{1}(\mathrm{UT}(\infty, R))=\mathrm{UT}(\infty, R)$ and $\gamma_{k}(\mathrm{UT}(\infty, R))=\mathrm{UT}(\infty, k-1, R)$ for $k>1$. Moreover, every element of the subgroup $\gamma_{k}(\mathrm{UT}(\infty, R))$ is a value of the basic commutator $c_{k}$. 
In fact it also follows from the Basic Theorem that all elements of subgroups $\mathrm{UT}(\infty, m, R)$ are values of the so-called Engel words $e_{m}$, each one defined only on two letters, where $e_{2}(x, y)=[x, y]$ and $e_{m+1}(x, y)=\left[e_{m}(x, y), y\right]$.

Corollary 1.2. If $R$ is an associative ring with 1 , such that $R^{*}$ is commutative, then every element of the subgroup $\gamma_{k}(\mathrm{UT}(\infty, R))$ is a value of Engel word $e_{k}$.

Then we discuss the implications of the Basic Theorem on the structure of the group of infinite triangular matrices $\mathrm{T}(\infty, R)$.

It is known that if $R=K$, where $K$ is a field of at least 3 elements, then $\mathrm{UT}(\infty, K)$ coincides with the derived subgroup $[\mathrm{T}(\infty, K), \mathrm{T}(\infty, K)]$ of $\mathrm{T}(\infty, K)$ [5]. An analogous equality holds for the groups of finitely dimensional matrices of size at least 3 by 3 : $[\mathrm{T}(n, K), \mathrm{T}(n, K)]=\mathrm{UT}(n, K)$ [23]. In our paper we extend this result to groups of matrices over a wider class of rings.

Since every diagonal entry of a triangular matrix from $\mathrm{T}(\infty, R)$ is invertible, simple computations show that the diagonal entries $D_{i, i}$ of the commutator $D=[A, B]$ of two upper triangular matrices $A$ and $B$ are

$$
D_{i, i}=A_{i, i}^{-1} B_{i, i}^{-1} A_{i, i} B_{i, i} .
$$

Hence, if $R^{*}$ is commutative, it follows that

$$
\begin{gathered}
{[\mathrm{T}(n, R), \mathrm{T}(n, R)] \subseteq \mathrm{UT}(n, R) \quad \text { for } \quad n \geq 2,} \\
{[\mathrm{~T}(\infty, R), \mathrm{T}(\infty, R)] \subseteq \mathrm{UT}(\infty, R) .}
\end{gathered}
$$

If we impose additional restriction on the ring $R$, we can prove the reverse inclusion.

Theorem 1.3. Let $R$ be an associative ring with 1 , such that $R^{*}$ is commutative and 1 is a sum of two invertible elements. Then $[\mathrm{T}(\infty, R), \mathrm{T}(\infty, R)]=$ $\mathrm{UT}(\infty, R)$ and every element of $\mathrm{UT}(\infty, R)$ is a product of at most two commutators.

Moreover the lower central series of the group $\mathrm{T}(\infty, R)$ is

$$
\gamma_{1}(\mathrm{~T}(\infty, R))=\mathrm{T}(\infty, R), \quad \gamma_{n}(\mathrm{~T}(\infty, R))=\mathrm{UT}(\infty, R), \quad \text { for } \text { all } n \geq 1,
$$

i.e it stabilizes on the group $\mathrm{UT}(\infty, R)$.

The condition that 1 is a sum of two invertible elements holds if, for example, $2 \in R^{*}$, because $1=2+(-1)$. It is clear that a ring with this property cannot have a two element field as a factor ring. For other properties of such rings we refer to $[24,25,26]$.

Then we discuss a more restrictive case of $R$ being a field and provide a representation of any (infinite) unitriangular matrix as a commutator of one diagonal and one unitriangular matrix in case the field is infinite. We also indicate some direct implications of Theorem 1 on the lower central series of the discussed groups of triangular matrices.

The Vershik-Kerov group $\mathrm{GL}_{V K}(\infty, R)$ is a group of all invertible infinite matrices with finite number of nonzero entries below the main diagonal (see 
$[8,6])$. Let $n$ be a natural number. $\operatorname{By} \operatorname{GL}(\infty, n, R)$ we denote the subgroup of $\mathrm{GL}_{V K}(\infty, R)$ consisting of all matrices of the form

$$
\left(\begin{array}{c|c}
G_{1} & G_{3} \\
\hline 0 & G_{2}
\end{array}\right)
$$

where $G_{1}$ is $n \times n$ invertible matrix from a general linear group $\mathrm{GL}(n, R), G_{2} \in$ $\mathrm{T}(\infty, R)$ and $G_{3}$ is arbitrary matrix of proper size. Note that the subgroups $\mathrm{GL}(\infty, n, R)$ form an ascending sequence, i. e. for all $n>1$ we have

$$
\mathrm{GL}(\infty, n, R) \subseteq \mathrm{GL}(\infty, n+1, R) .
$$

It is clear that

$$
\mathrm{GL}_{V K}(\infty, R)=\bigcup_{n>1} \mathrm{GL}(\infty, n, R) .
$$

For a commutative ring $R$ by $\mathrm{SL}_{V K}(\infty, R)$ we denote the subgroup of $\mathrm{GL}_{V K}(\infty, R)$ consisting of all matrices of the form (1), where $G_{1}$ is a matrix from the special linear group $\mathrm{SL}(n, R)(n \in \mathbb{N})$, and $G_{2} \in \mathrm{UT}(\infty, R)$.

Compiling Theorem 1 with results of [6], we characterize the derived subgroup of the Vershik-Kerov group and improve the upper bound of the commutator width of this group.

Theorem 1.4. Let $K$ be a field such that $|K|>3$. Then the commutator subgroup of the group $\mathrm{GL}_{V K}(\infty, K)$ coincides with the group $\mathrm{SL}_{V K}(\infty, K)$ and every element of $\mathrm{SL}_{V K}(\infty, K)$ is a product of at most 2 commutators.

Our proofs for the groups of infinite matrices over $R$ may be directly reformulated for the respective groups of finite matrices over $R$. In particular we have:

Theorem 1.5. Let $R$ be an associative ring with 1 , such that $R^{*}$ is commutative. Then

i) $\gamma_{k}(\mathrm{UT}(n, R))=\mathrm{UT}(n, k, R)$ and every element of $\gamma_{k}(\mathrm{UT}(n, R))$ is a value of the basic commutator $c_{k}$.

ii) Every element of $\gamma_{k}(\mathrm{UT}(n, R))$ is a value of the Engel word $e_{k}$.

Moreover, if 1 is a sum of two invertible elements, then

iii) $[\mathrm{T}(n, R), \mathrm{T}(n, R)]=\mathrm{UT}(n, R)$ whenever $n \geq 2$.

iv) Every element of $\gamma_{2}(\mathrm{~T}(2, R))=\mathrm{UT}(2, R)$ is a commutator.

v) Every element of $\gamma_{2}(\mathrm{~T}(n, R))=\mathrm{UT}(n, R)$ with $n>2$ is a product of at most two commutators.

The remaining part of the paper is organized in two sections. In the first section we give the detailed proof of the Main Theorem. Then in the other one we discuss the implications of the Basic Theorem and prove the corollaries and theorems, stated in the Introduction. 


\section{Proof of the Basic Theorem}

For given $m \geq 1$ and a matrix $C \in \mathrm{UT}(\infty, m, R)$ we prove the result by calculating the entries of the required matrix $A \in \mathrm{UT}(\infty, m-1, R)$, for which $C=[A, B]$. We find the entries of $A$ employing induction on its columns.

We first rewrite the desired equality $C=[A, B]$ as $C A=B^{-1} A B$ and compare the matrices on both sides entrywise:

$$
(C A)_{i, j}=\left(B^{-1} A B\right)_{i, j}, \quad i<j .
$$

We denote for brevity $L_{i, j}=(C A)_{i, j}$ and $P_{i, j}=\left(B^{-1} A B\right)_{i, j}$. Then, keeping in mind that $B^{-1}=\mathbf{1}_{\infty}+\sum_{n=1}^{\infty} \mathbf{1}_{i, i+1}$ we have:

$$
\begin{aligned}
L_{i, i+k} & =A_{i, i+k}+C_{i, i+k}+\sum_{s=1}^{k-1} A_{i, i+s} C_{i+s, i+k}, \\
P_{i, i+k} & =\sum_{t=0}^{k} A_{i, i+t} B_{i+t, i+k}+\sum_{t=1}^{k} A_{i+1, i+t} B_{i+t, i+k}, \\
L_{i, i+k} & =P_{i, i+k} .
\end{aligned}
$$

Direct calculations show that the entry $A_{i, i+k}$ reduces on both sides of the equation (2). However from (2) for $k>1$ we may determine:

$$
A_{i+1, i+k}=C_{i, i+k}+\sum_{s=1}^{k-1} A_{i, i+s} C_{i+s, i+k}-\sum_{t=0}^{k-1} A_{i, i+t} B_{i+t, i+k}+\sum_{t=1}^{k-1} A_{i+1, i+t} B_{i+t, i+k},
$$

and it is clear that every entry $A_{i+1, j}, j>i+1$, is computable whenever the matrices $B, C$ and all entries $A_{i, j}$ and $A_{i+1, j}$ for $j<i+k$ are known.

For a particular solution to $C=[A, B]$ we have to choose the first row of $A$. In fact an arbitrary choice would provide a solution, however for the statement of our theorem we need to find $A$ in $\mathrm{UT}(\infty, m-1, R)$. Thus we set $A_{1, j}=0$ for all $1<j \leq m$ and choose all other entries $A_{1, j}, j>m$ arbitrarily. Assume now that $A_{i, i+k}=0$ for all $i \leq n$ and $0<k<m$. Then using (3) with $i=n$ for $k<m$ we have:

$$
A_{n+1, n+k}=C_{n, n+k}+\sum_{s=1}^{k-1} A_{n, n+s} C_{n+s, n+k}-\sum_{t=0}^{k-1} A_{n, n+t} B_{n+t, n+k}+\sum_{t=1}^{k-1} A_{n+1, n+t} B_{n+t, n+k} .
$$

Recall that $C_{n, n+k}=0$ for all $k \leq m$. In the case of $k>1$ we have:

$$
A_{n+1, n+k}=-\sum_{t=1}^{k-1} A_{n, n+t} B_{n+t, n+k}-\sum_{t=2}^{k-1} A_{n+1, n+t} B_{n+t, n+k},
$$

and in particular we recursively find the entries:

$$
\begin{aligned}
A_{n+1, n+2} & =-A_{n, n+1} B_{n+1, n+2}=0 \\
A_{n+1, n+3} & =-A_{n, n+1} B_{n+1, n+3}-A_{n, n+2} B_{n+1, n+3}-A_{n+1, n+2} B_{n+2, n+3}=0 \\
& \cdots \\
A_{n+1, n+m} & =-\sum_{t=1}^{k-1} A_{n, n+t} B_{n+t, n+k}-\sum_{t=2}^{k-1} A_{n+1, n+t} B_{n+t, n+k}=0 .
\end{aligned}
$$


By induction we have $A \in \mathrm{UT}(\infty, m-1, R)$ as desired, and we find every entry of the infinite matrix $A$ in finitely many steps. Thus, the theorem follows.

We also note that if $C$ is not contained in $\mathrm{UT}(\infty, m+1, R)$, then we cannot choose $A$ in $\mathrm{UT}(\infty, m, R)$, as in this case the commutator $[A, B]$ lies in $\mathrm{UT}(\infty, m+1, R)$.

\section{Implications of the Basic Theorem}

We start with the direct implications of the Basic Theorem on the structure of the lower central series of the group $\mathrm{UT}(\infty, R)$. Namely, since every matrix from $\mathrm{UT}(\infty, m, R)$ is a commutator of a matrix $B$ from $\mathrm{UT}(\infty, R)$ and a matrix from $\mathrm{UT}(\infty, m-1, R)$ then we have the following inclusion:

$$
\mathrm{UT}(\infty, m, R) \subseteq[\mathrm{UT}(\infty, m-1, R), \mathrm{UT}(\infty, R)] .
$$

As the inverse inclusion is obvious, we obtain the statement of Corollary 1. Moreover, if $A$ is an arbitrary matrix from $\gamma_{m}(\mathrm{UT}(\infty, R))=\mathrm{UT}(\infty, m, R)$ then by the Basic Theorem

$$
A=\left[A_{1}, B\right],
$$

where $A_{1} \in \mathrm{UT}(\infty, m-1, R)$. Now, applying again the Main theorem to the matrix $A_{1}$ we write

$$
A=\left[A_{1}, B\right]=\left[\left[A_{2}, B\right], B=\left[A_{2}, B, B\right], \quad A_{2} \in \mathrm{UT}(\infty, m-2, R) .\right.
$$

We repeat this reasoning by choosing consequently the matrices $A_{i} \in \mathrm{UT}(\infty, m-$ $i, R)$ and finally we obtain:

$A=\left[A_{1}, B\right]=\left[A_{2}, B, B\right]=\left[A_{3}, B, B, B\right]=\ldots=\left[A_{m}, B, B, \ldots, B\right]=e_{m+1}\left(A_{m}, B\right)$

with $A_{m} \in \mathrm{UT}(\infty, R)$. We have shown that every matrix in $\mathrm{UT}(\infty, m, R)$ is a value of the Engel word $e_{m+1}$ as stated in Corollary 2 .

\subsection{Proof of Theorem 1}

Let $R$ be an associative ring with unity 1 , such that $R^{*}$ is commutative and the unity can be decomposed to a sum of two invertible elements: $1=\beta+\gamma$, where $\beta, \gamma \in R^{*}$.

We follow the ideas of Theorem 2 in [6].

Let $A=\left(a_{i j}\right) \in \mathrm{UT}(\infty, R)$. We put

$$
C=\mathbf{1}_{\infty}+\sum_{i=1}^{\infty}(-1)^{i} a_{i, i+1} \beta^{-1} \gamma^{(i+1)} \bmod 2 \mathbf{1}_{i, i+1}
$$

and a diagonal matrix

$$
D=\sum_{i=1}^{\infty} \gamma^{(i+1)} \quad \bmod ^{2} \mathbf{1}_{i, i}
$$


We observe that if $[C, D]=U=\left(u_{i j}\right)$ then direct calculations show that $u_{i, i+1}=a_{i, i+1}$ for all $i \in \mathbf{N}$. Hence,

$$
A=U \cdot\left(U^{-1} A\right),
$$

where $U^{-1} A \in \mathrm{UT}(\infty, 1, R)$ and by the Basic Theorem $U^{-1} A$ is a commutator. Thus $A$ is a product of two commutators contained in $[\mathrm{UT}(\infty, R), \mathrm{T}(\infty, R)]$ and we have the following inclusions:

$$
[\mathrm{T}(\infty, R), \mathrm{T}(\infty, R)] \supseteq[\mathrm{UT}(\infty, R), \mathrm{T}(\infty, R)] \supseteq \mathrm{UT}(\infty, R) .
$$

As the reverse inclusion $[\mathrm{T}(\infty, R), \mathrm{T}(\infty, R)] \subseteq \mathrm{UT}(\infty, R)$ is obvious, we have

$$
[\mathrm{T}(\infty, R), \mathrm{T}(\infty, R)]=\mathrm{UT}(\infty, R),
$$

and the lower central series of $\mathrm{T}(\infty, R)$ is

$$
\begin{aligned}
& \gamma_{1}(\mathrm{~T}(\infty, R))=\mathrm{T}(\infty, R) \\
& \gamma_{i}(\mathrm{~T}(\infty, R))=\left[\gamma_{i-1}(\mathrm{~T}(\infty, R)), \mathrm{T}(\infty, R)\right]=\mathrm{UT}(\infty, R), \quad \text { for } i>1 .
\end{aligned}
$$

This completes the proof of Theorem 1 .

We note that if $R=K$, where $K$ is a field, one can prove a stronger result that every infinite unitriangular matrix $A \in \mathrm{UT}(\infty, K)$ is a commutator of two infinite triangular matrices (see [5]). Given a unitriangular matrix, one can construct triangular matrices, such that their commutator is equal to the given matrix. However, this construction does not allow for none of these matrices to be fixed. We prove that if $K$ is infinite, one can fix one of the matrices by giving another solution to the commutator equation in $\mathrm{T}(\infty, K)$.

Theorem 3.1. Let $K$ be an infinite field. Then there exists an infinite diagonal matrix $D$ with pairwise distinct diagonal entries such that every unitriangular matrix $A \in \mathrm{UT}(\infty, K)$ is commutator of $D$ and another unitriangular matrix.

Proof. We first note that since $K$ is infinite, then there exists an infinite diagonal matrix $D$ with pairwise distinct diagonal entries. We fix $D$ arbitrarily. Now we construct a unitriangular matrix $U \in \mathrm{UT}(\infty, K)$, such that $A=[U, D]$. The construction is inductive with respect to the consecutive columns of $U$.

Given an infinite triangular matrix $A \in \mathrm{T}(\infty, R)$ we will denote its top-left corner block by $A(n)$. Obviously $A(n) \in \mathrm{T}(n, R)$.

We start with $A(2)=\mathbf{1}_{2}+a \mathbf{1}_{1,2}, a \in K$ and put $U(2)=\mathbf{1}_{2}+u \mathbf{1}_{1,2} \in$ $U T_{n}(K)$, such that $u=\left(D_{1,1}^{-1} D_{2,2}-1\right)^{-1} a$. Direct calculations show that $A(2)=$ $[U(2), D(2)]$.

Now, let us assume that for a given $n$ we found $U(n)$ such that $A(n)=$ $[U(n), D(n)]$. Consider matrix $A(n+1) \in U T_{n+1}(K)$ :

$$
a(n+1)=\left(\begin{array}{cc}
A(n) & \underline{a} \\
\underline{0} & 1
\end{array}\right),
$$


where $A(n) \in U T(n, K), \underline{a}^{T}=\left(a_{1}, a_{2}, \ldots, a_{n}\right) \in K^{n}$ and $\underline{0} \in K^{n}$ is a zero vector. We define $U(n+1) \in U T(n+1, K)$ as follows:

$$
U(n+1)=\left(\begin{array}{cc}
U(n) & \underline{u} \\
\underline{0} & 1
\end{array}\right),
$$

where $U(n)$ is a matrix determined in the inductive assumption an $\underline{u}^{T}=$ $\left(u_{1}, u_{2}, \ldots, u_{n}\right) \in K^{n}$. Then we have:

$$
[U(n+1), D(n+1)]=\left(\begin{array}{cc}
{[U(n), D(n)]} & U(n)^{-1}\left(D(n)^{-1} D_{n+1, n+1}-\mathbf{1}_{n}\right) \underline{u} \\
\underline{0} & 1
\end{array}\right),
$$

and for the equality $A(n+1)=[U(n+1), D(n+1)]$ it suffices that

$$
\underline{a}=U^{-1}(n)\left(D^{-1}(n) D_{n+1, n+1}-\mathbf{1}_{n}\right) \underline{u} .
$$

By the assumptions on matrix $D$ the matrix $D^{-1}(n) D_{n+1, n+1}$ is invertible and so is the matrix $U^{-1}(n)\left(D^{-1}(n) D_{n+1, n+1}-\mathbf{1}_{n}\right)$. Hence we may put

$$
\underline{u}=\left(U^{-1}(n)\left(D^{-1}(n) D_{n+1, n+1}-\mathbf{1}_{n}\right)\right)^{-1} \underline{a} .
$$

Then $A(n+1)=[U(n+1), D(n+1)]$ as desired. Hence we may inductively compute every entry of the infinite matrix $A$ in finitely many steps and the theorem follows.

From Theorem 4 it follows immediately:

Corollary 3.2. If $K$ is an infinite field then every infinite unitriangular matrix is an $m$-Engel word in $\mathrm{T}(\infty, K)$ for every $m \geq 2$.

The results discussed above can be applied also to improve the statements of Theorem 1 in [6]. For the proof of the next theorem we assume that $R=K$ is a field.

\subsection{Proof of Theorem 2}

Since $\mathrm{GL}_{V K}(\infty, K)$ is a sum of the ascending chain of subgroups $\mathrm{GL}(\infty, n, K)$, then for every two matrices $A, B \in \mathrm{GL}_{V K}(\infty, K)$ we find $n$ such that $A, B \in$ $G L(\infty, n, K)$. Let

$$
A=\left(\begin{array}{c|c}
G_{1} & G_{3} \\
\hline 0 & G_{2}
\end{array}\right), \quad B=\left(\begin{array}{c|c}
H_{1} & H_{3} \\
\hline 0 & H_{2}
\end{array}\right) .
$$

Then

$$
[A, B]=\left(\begin{array}{c|c}
{\left[G_{1}, H_{1}\right]} & G_{4} \\
\hline 0 & {\left[G_{2}, H_{2}\right]}
\end{array}\right),
$$

where $\left[G_{1}, H_{1}\right] \in \mathrm{SL}(n, K),\left[G_{2}, H_{2}\right] \in \mathrm{UT}(\infty, K)$ and $G_{4}$ is a matrix of respective dimensions. This clearly implies the inclusion

$$
\left[\mathrm{GL}_{V K}(\infty, K), \mathrm{GL}_{V K}(\infty, K)\right] \subseteq \mathrm{SL}_{V K}(\infty, K) .
$$


Now we prove the reverse inclusion. Simple calculations show that every matrix in $\mathrm{SL}_{V K}(\infty, K)$ can be decomposed as a product:

$$
\left(\begin{array}{c|c}
G_{1} & G_{3} \\
\hline 0 & G_{2}
\end{array}\right)=\left(\begin{array}{c|c}
\mathbf{1}_{n} & G_{3} \\
\hline 0 & G_{2}
\end{array}\right)\left(\begin{array}{c|c}
G_{1} & 0 \\
\hline 0 & \mathbf{1}_{\infty}
\end{array}\right)
$$

In [27] it was shown (Theorems 1 and 2) that in the case $K \neq \mathbb{F}_{2}, \mathbb{F}_{3}$, then every element of $\mathrm{SL}(n, K)$ is a commutator. It is clear that this statement can be generalized to all infinite matrices of the form

$$
\left(\begin{array}{c|c}
G_{1} & 0 \\
\hline 0 & \mathbf{1}_{\infty}
\end{array}\right)
$$

where $G_{1} \in \mathrm{SL}(n, K)$. Hence there exist matrices $H_{1}, H_{2} \in \mathrm{GL}(n, K)$ such that:

$$
\left(\begin{array}{c|c}
G_{1} & 0 \\
\hline 0 & \mathbf{1}_{\infty}
\end{array}\right)=\left[\left(\begin{array}{c|c}
H_{1} & 0 \\
\hline 0 & \mathbf{1}_{\infty}
\end{array}\right),\left(\begin{array}{c|c}
H_{2} & 0 \\
\hline 0 & \mathbf{1}_{\infty}
\end{array}\right)\right] .
$$

Moreover, from [5] we have that

$$
\left(\begin{array}{c|c}
\mathbf{1}_{n} & G_{3} \\
\hline 0 & G_{2}
\end{array}\right)
$$

is a commutator. Thus

$$
\left(\begin{array}{c|c}
G_{1} & G_{3} \\
\hline 0 & G_{2}
\end{array}\right)
$$

is a product of at most two commutators and

$$
\mathrm{SL}_{V K}(\infty, K) \subseteq\left[\mathrm{GL}_{V K}(\infty, K), \mathrm{GL}_{V K}(\infty, K)\right] .
$$

This completes the proof.

\subsection{Corollaries}

Due to the natural embeddings of the groups $\mathrm{UT}(n, R)$ and $\mathrm{T}(n, R)$ into $\mathrm{UT}(\infty, R)$ and $\mathrm{T}(\infty, R)$, the statements of Theorem 3 follow directly from calculations performed in proofs of Theorems 1 and 2 .

It is worth mentioning that Theorem 3 generalizes the results on the groups of finite dimensional unitriangular matrices and finite dimensional triangular matrices over a field, presented in [12] and [16] to the case of respective matrix groups over any associative ring $R$ with unity such that the group $R^{*}$ is commutative. This generalization cannot be improved further.

\subsection{References}

\section{References}

[1] E. Asplund, Inverses of matrices $\left\{a_{i, j}\right\}$ which satisfy $a_{i, j}=0$ for $j>i+p$, Math. Scand. 7(1959), p. $57-60$ 
[2] W. Hołubowski, An inverse matrix of an upper triangular matrix can be lower triangular, Discuss. Math. General Algebra and App. (2002), 22, p. $161-166$

[3] R. Słowik, On one property of normal subgroups of $\mathrm{UT}_{\infty}(R)$, Linear Algebra and its Applications 437 (2012), p. 2300-2307

[4] R. Słowik, The lower central series of subgroups of the Vershik-Kerov group, Linear Algebra and its Applications 436, 7 (2012), p.2299-2310

[5] R. Słowik, Bijective maps of infinite triangular and unitriangular matrices preserving commutators, Linear and Multilinear Algebra, DOI:10.1080/03081087.2012.728214

[6] C. K. Gupta, W. Hołubowski, Commutator subgroup of Vershik-Kerov group, Linear Algebra and its Applications 436, 11 (2012), p.4279-4284

[7] V. Snaith, The upper triangular group and operations in algebraic Ktheory, Topology 41 (2002), no. 6, 1259-1275.

[8] A. M. Vershik, S. V. Kerov, On an infinite-dimensional group over a finite field, Funct. Anal. Appl. 32 (1998), no. 3, 147-152.

[9] A. M. Vershik, S. V. Kerov, Four drafts on the representation theory of the group of infinite matrices over a finite field, J. Math Sci. (N.Y.) 147 (2007), no. $6,7129-7144$.

[10] V. Bardakov, A. Vesnin, M.K. Yadav, Class preserving automorphisms of unitriangular groups, Internat. J. Algebra Comput. 22 (2012), no.3, 17pp.

[11] A. Bier, On Solvability of Engel Equations In the Group of Triangular Matrices Over a Field, Linear Algebra and its Applications (2012) DOI: 10.1016/j.laa.2012.10.009

[12] A. Bier, The width of verbal subgroups in groups of unitriangular matrices over a field, Int. J. Alg. Comput. 22, 3 (2012), 1250019, 20 pp.

[13] F. Kuzucuoglu, Isomorphisms of the unitriangular groups and associated Lie rings for the exceptional dimensions, Acta Appl. Math. 85 (2005), no.13, 2009-213.

[14] T.Le, Counting irreducible representations of large degree of the upper triangular groups, J. Algebra 324 (2010), no. 8, 1803-1817.

[15] M. Loukaki, Counting characters of small degree of upper triangular groups, J. Pure Appl. Algebra 215 (2011), no.2, 154-160.

[16] Yu. V. Sosnovsky, On the width of verbal subgroups of the groups of triangular matrices over a field of arbitrary characteristic, arXiv:1201.6513v1 [math.GR] 
[17] J.Cohen, K.Koh, The group of units in a compact ring, J. Pure Appl. Algebra 54 (1988), no.2, 167-179.

[18] K. E. Eldridge, I. Fisher, D.C.C. rings with a cyclic group of units, Duke Math. J. 34 (1967), 243-248.

[19] D. Khurana, G. Marks, A. K. Srivastava, On Unit-central rings, Advances in ring theory, Trend in Mathematics, Springer 2010, 205-212.

[20] W.K. Nicholson, H. J. Springer, Commutativity of rings with abelian or solvable units, Proc. Amer. Math. Soc. 56 (1976), no. 1, 59-62.

[21] W. K. Nicholson, Semiperfect rings with abelian group of units, Pacific J. Math. 49 (1973), 191-198.

[22] D.J.S. Robinson, A course in the theory of groups, Springer-Verlag, New York 1982.

[23] M. I. Kargapolov, Yu. I. Merzljakov, The fundamentals of group theory, Springer-Verlag, New York 1979.

[24] M. Henriksen, Two classes of rings generated by their units, J. Algebra 31, (1974), 182-193.

[25] D.Khurana, A.K. Srivastava, Right self-injective rings in which each element is sum of two units, J. Algebra and its Appl. 6 (2007), no. 2, 281-286.

[26] R. Raphael, Rings which are generated by thei units, J. Algebra 28 (1974), 199-205.

[27] R.C. Thompson, Commutators in the special and general linear groups, Trans. Amer. Math. Soc. 101 (1961) no. 1, 16-33. 